\title{
Optimal Location of FACTS Devices for Congestion Management in Deregulated Power Systems
}

\author{
K.Vijayakumar \\ SRM University \\ Kattankulathur, Chennai
}

\begin{abstract}
In the emerging deregulated electric power market, congestion management becomes extremely important and it can impose barrier to electric power trading. There are two types of congestion management methodologies to relieve congestion in transmission lines. One is non-cost free methods and another is cost-free methods, among them later method relieves the congestion technically whereas the former is related more with the economics. In this paper congestion is relieved using cost free method. Among the various cost free methods, use of FACTS devices method is considered in this paper. The optimal location of TCSC and UPFC to relieve congestion in the network is proposed. In congestion management, the objective function is nonlinear hence for solving this function genetic algorithm (GA) technique is used. The above method is tested on IEEE 57-bus system and it can be readily extended to any practical systems.
\end{abstract}

\section{General Terms}

Congestion, Deregulated Power System, Flexible AC Transmission Systems (FACTS), Thyristor Controlled Series Capacitor (TCSC), Unified Power Flow Controller (UPFC), Genetic Algorithms(GA), Optimal Power Flow (OPF)

\section{Keywords}

FACTS, Unified Power Flow Controller (UPFC), Genetic Algorithm (GA) Deregulation, Optimal Power Flow (OPF) .

\section{INTRODUCTION}

The restructuring in electric power sector has lead to larger use of transmission grids. In deregulated power market, the power system is operated almost to its rated capacity all the times. Congestion may occur in transmission line due to lack of coordination between generation and transmission utilities. So congestion management becomes very essential in deregulated power systems. In regulated power system Transmission Companies (TRANSCOs), Generation Companies (GENCOs) and Distribution Companies (DISCOs) all come under one organization, generally government. Whatever the expenditure incurred on power system will be bared by the government and at the same whatever revenue came it will go to government. On the other hand in deregulated power systems TRANSCOs, GENCOs, DISCOs are under different organizations [1]-[3]. To maintain the coordination between them there will be one system operator in all types of deregulated power system models, generally it is Independent System Operator (ISO). In deregulated environment all the GENCOs and DISCOs make the transactions ahead of time, but by the time of implementations there may be congestion in some of the transmission lines. Hence ISO has to relieve that congestion so that the system is maintained in secure state. To relieve the congestion ISO can use mainly two types of techniques which are as follows [4]-[6]:

\section{A.Cost-free means}

(i) Out-aging of congested lines.

(ii) Operation of transformer taps/phase shifters.

(iii) Operation of FACTS devices particularly series devices.

\section{B. Non-cost-free means:}

(i) Re-dispatch of generation in a manner different from the natural settling point of the market. Some generators back down while others increase their output. The effect of this is that generators no longer operate at equal incremental costs.

(ii) Curtailment of loads and the exercise of (not-cost-free) load interruption options.

Among the above two main techniques cost-free means have the advantages like it is not going to affect economical matters, so to relieve the congestion GENCOs and DISCOs will not come into picture. In this paper, FACTS devices are used to relieve the congestion because they posses many advantages as compared with the other techniques [7]-[13].

In congestion management the objective function and constraints are nonlinear and non-convex. To solve such equations classical techniques offer good results but with a slow convergence ratio and not always giving the optimal solution. Genetic algorithms are also being applied to a wide range of optimization and learning problems in many domains. Genetic algorithms lend themselves well to power system optimization, and can offer significant advantages in a solution methodology and optimization performance.

The rest of the paper is organized as follows: Optimal Power Flow (OPF) problem is formulated for relieving congestion management in Section 2, Section 3 describes genetic algorithm technique. In section4, Static modeling of TCSC and UPFC is discussed. Section 5, describes about optimal locations of TCSC and UPFC. In section 6, results and discussions are presented and finally the paper is concluded with section 7 .

\section{PROBLEM FORMULATION FOR CONGESTION MANAGEMENT}

The basic principle for the transmission congestion management could be illustrated with the help of the traditional spot pricing theory. In this framework, the 
central dispatcher optimally dispatches the generators such that the social welfare is maximized while satisfying the operation and security related constraints. Specifically, the dispatcher solves the following optimization problem to maximize the social welfare:

$\min \left(\sum_{i=1}^{N_{G}} C_{G_{i}}\left(P_{G_{i}}\right)-\sum_{i=1}^{N_{D}} B_{D_{i}}\left(P_{D_{i}}\right)\right.$

Subjected to

$$
\begin{gathered}
P_{g i}-P_{d i}=\sum_{j=1}^{N_{B}}\left|V_{i}\right|\left|V_{j}\right|\left|Y_{i j}\right| \cos \left(\delta_{i}-\delta_{j}-\theta_{i j}\right) \\
Q_{g i}-Q_{d i}=\sum_{j=1}^{N_{B}}\left|V_{i}\right|\left|V_{j}\right|\left|Y_{i j}\right| \sin \left(\delta_{i}-\delta_{j}-\theta_{i j}\right) \\
P_{g i, \min } \leq P_{g i} \leq P_{g i, \max } \\
Q_{g i, \min } \leq Q_{g i} \leq Q_{g i, \max } \\
P_{d i, \min } \leq P_{d i} \leq P_{d i, \max } \\
Q_{d i, \min } \leq Q_{d i} \leq Q_{d i, \max } \\
\quad V_{i, \min } \leq V_{i} \leq V_{i, \text { max }}
\end{gathered}
$$

$T_{i j} \geq 0$

Where, $\mathrm{PGi}_{\mathrm{G}}$, QGi are the real and reactive power generation at bus i. $\mathrm{P}_{\mathrm{di}}, \mathrm{Q}_{\mathrm{di}}$ are the real and reactive power demands at bus i.

$\mathrm{V}_{\mathrm{i}}, \delta_{\mathrm{i}}$ are voltage and angles at bus $\mathrm{i}$.

$P_{\text {gi,min, }} P_{\text {gi,max }}$ real power minimum and maximum generation limits at bus $i$.

Qgi,min, Qgi,max reactive power minimum and maximum generation limits at bus $i$.

$\mathrm{P}_{\mathrm{di}, \min }, \mathrm{P}_{\mathrm{di}, \max }$ real power minimum and maximum demand limits at bus $i$.

Qdi,min, Qdi,max reactive power minimum and maximum demand limits at bus $i$.

In the objective function $\mathrm{C}_{\mathrm{Gi}} \quad\left(\mathrm{P}_{\mathrm{Gi}}\right)$ is cost function for generating real power $\mathrm{P}_{\mathrm{Gi}}$ at bus $\mathrm{i}, \mathrm{B}_{\mathrm{Di}}\left(\mathrm{P}_{\mathrm{Di}}\right)$ and is the demand function. $T_{i j}$ is the bilateral transaction between supplier at node $\mathrm{i}$ and consumer at node $\mathrm{j}$.

By solving above optimization problem the generation schedule is be obtained and with this schedule the line flows are found. Then line flows are checked whether within the maximum limits are not, if any of the line exceeds the thermal limit is said to be congested and it has to be relieved. To solve the above optimization problem classical techniques suffers from the local optima and they need auxiliary information about the objective function [14]. By heuristic search method global optima can be found. Among the heuristic search methods genetic algorithm (GA) is one of the good techniques [15] and it is used in this paper.

\section{GENETIC ALGORITHM}

Genetic algorithms are one of the best ways to solve a complex optimization problem. GAs are very general algorithm and can work well in any search space. Genetic algorithm will be able to produce a high quality solution. Genetic algorithms use the principles of selection and evolution to produce several solutions to a given problem. Genetic algorithms tend to thrive in an environment in which there is a very large set of candidate solutions and in which, the search space is uneven and has many hills and valleys. True, genetic algorithms will do well in any environment, but they will be greatly outclassed by more situation specific algorithms in the simpler search spaces. They are, however, one of the most powerful methods with which to (relatively) quickly create high quality solutions to a problem.

The most common type of genetic algorithm works like this: a population is created with a group of individuals created randomly. The individuals in the population are then evaluated. The evaluation function is provided by the programmer and gives the individuals a score based on how well they perform at the given task. Two individuals are then selected based on their fitness, the higher the fitness, higher the chance of being selected. These individuals then "reproduce" to create one or more offspring, after which the offspring are mutated randomly. This continues until a suitable solution has been found or a certain number of generations have passed, depending on the needs of the problem.

Each GA includes several major operations [6]: (i)selection, (ii)crossover and (iii) mutation. The selection operator picks up the best solutions from the population, thus pushing the search towards optimum. The crossover operation combines two members of the population and generates two offspring. The mutation operator randomly generates a point, thus exploring all regions of the search space

Parent selection is a simple procedure where by two chromosomes are selected from the parent population based on their fitness value. Solutions with high fitness values have a high probability of controlling new offspring to the next generation. The selection rule used in this paper is a simple roulette-wheel selection.

Crossover is an extremely important operator for the GA. It is responsible for the structure recombination (information exchange between mating chromosomes) and the convergence speed of the GA and is usually applied with high probability (0.6 -0.9). The chromosomes of the two parents selected are combined to form new chromosomes that inherit segments or information stored in parent chromosomes. Until now, many crossover schemes, such as single point, multipoint, or uniform crossover have been proposed in the literature. Single point crossover has been used in this paper.

Mutation is the operator responsible for the injection of new information. With a small probability, random bits of the offspring chromosomes flip from 0 to 1 and vice versa and give new characteristics that do not exist in the parent population. In this paper, the mutation operator is applied with a relatively small probability $(0.001-0.005)$ to 
every bit of the chromosome. The FF evaluation and genetic evolution take part in an iterative procedure, which ends when a maximum number of generations are reached.

\section{GENETIC ALGORITHM}

If load flows are performed with the optimal schedule obtained from OPF, few of the line flows may exceeds line limits. ISO has to relieve such congestion in the lines to maintain the system in secure state. FACTS technology is improved day by day because their flexible control and versatility. Some of the advantages of FACTS devices are [18]:

- Greater control of power, so that it flows on prescribed transmission routes.

- Secure loading of transmission lines to level nearer their thermal limits.

- Greater ability of transfer between controlled areas.

- Prevention of cascading outages.

- Damping of power system oscillations.

Among the FACTS devices Thyristor Controlled Series Capacitor (TCSC) and Unified Power Flow Controller (UPFC) are versatile devices and in this paper they are used to relieve the congestion [16]-[22].

\subsection{Modelling of Transmission line}

Fig. 1 shows a simple transmission line represented by its lumped $\Pi$ - equivalent parameters connected between bus- $i$ and bus- $j$.

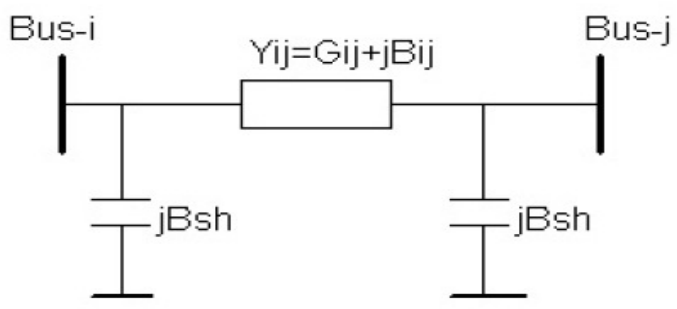

Figure 1 Model of transmission line.

Let complex voltages at bus- $i$ and bus- $j$ are $V_{i} \quad\left\llcorner\delta_{i}\right.$ and $V_{j} \quad L \delta_{j}$ respectively. The real and reactive power flow from bus- $i$ and bus- $j$ ( $\mathrm{P}_{\mathrm{ij}}$ and $\mathrm{Q}_{\mathrm{ij}}$ ) can be written as:

$$
\begin{gathered}
P_{i j}=V_{i}^{2} G_{i j}-V_{i} V_{j}\left[G_{i j} \cos \left(\delta_{i j}\right)+B_{i j} \sin \left(\delta_{i j}\right)\right] \\
Q_{i j}=-V_{i}^{2}\left(B_{i j}+B_{s h}\right)-V_{i} V_{j}\left[G_{i j} \sin \left(\delta_{i j}\right)-B_{i j} \cos \left(\delta_{i j}\right)\right]
\end{gathered}
$$

Similarly the real and reactive power flow from bus- $j$ and bus- $i\left(\mathrm{P}_{\mathrm{ji}}\right.$ and $\left.\mathrm{Qji}\right)$ can be written as

$$
\begin{array}{r}
P_{j i}=V_{j}^{2} G_{i j}-V_{i} V_{j}\left[G_{i j} \cos \left(\delta_{i j}\right)-B_{i j} \sin \left(\delta_{i j}\right)\right] \\
Q_{j i}=-V_{j}^{2}\left(B_{i j}+B_{s h}\right)+V_{i} V_{j}\left[G_{i j} \sin \left(\delta_{i j}\right)+B_{i j} \cos \left(\delta_{i j}\right)\right]
\end{array}
$$

\subsection{Modelling of TCSC}

The effect of FACTS devices like TCSC on the network can be seen as a controllable reactance inserted in the related transmission line. The model of the network with TCSC is shown in Fig. 2.

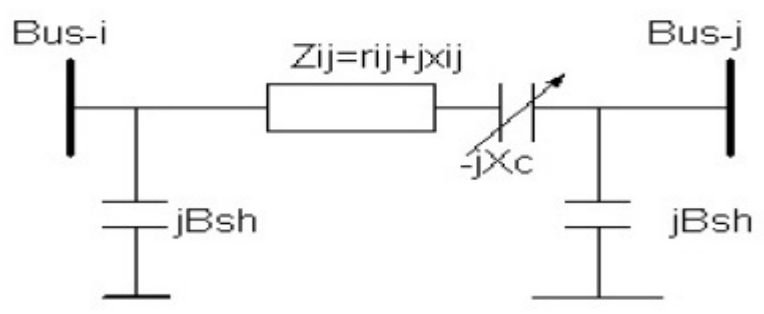

Figure 2 Modelling of transmission line with TCSC

During steady state the TCSC can be considered as a static capacitor/reactor offering impedance jXTCSC . The controllable reactance XTCSC is directly used as a control variable to be implemented in the power flow equations. The real power and reactive power flow equation of the branch $k$ flowing from bus $i$ to $j$ can be expressed as:

$$
\begin{gathered}
P_{i j}^{c}=V_{j}^{2} G_{i j}^{c}-V_{i} V_{j}\left[G_{i j}^{c} \cos \left(\delta_{i j}\right)+B_{i j}^{c} \sin \left(\delta_{i j}\right)\right](12) \\
Q_{i j}^{c}=-V_{i}^{2}\left(B_{i j}^{c}+B_{s h}\right)-V_{i} V_{j}\left[G_{i j}^{c} \sin \left(\delta_{i j}\right)-B_{i j}^{c} \cos \left(\delta_{i j}\right)\right](13) \\
Q_{j i}^{c}=-V_{j}^{2}\left(B_{i j}^{c}+B_{s h}\right)+V_{i} V_{j}\left[G_{i j}^{c} \sin \left(\delta_{i j}\right)+B_{i j}^{c} \cos \left(\delta_{i j}\right)\right](14) \\
\text { Where } G_{i j}^{c}=\frac{r_{i j}}{r_{i j}^{2}+\left(x_{i j}-x_{T C S C}\right)^{2}} \text { and } B_{i j}^{c}=\frac{-\left(x_{i j}-x_{T C S C}\right)}{r_{i j}^{2}+\left(x_{i j}-x_{T C S C}\right)^{2}}
\end{gathered}
$$

The change in the line flows due to series capacitance can be represented as a line without series capacitance with power injected at the receiving and sending ends of the line as shown in Fig. 3. The real power injections at bus- $i$ (Pic) and bus- $j$ (Pjc) can be expressed, by subtracting eqn. (8) from eqn. (12) and vice versa.

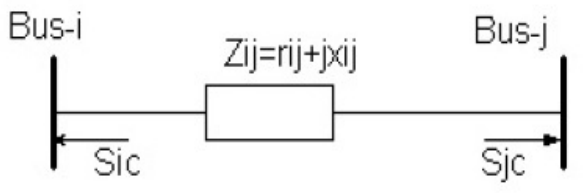

Figure 3 Injection model of TCSC 


\subsection{Modelling of UPFC}

Unified Power Flow Controller (UPFC) was devised for realtime control and dynamic compensation of ac transmissions system, providing multifunctional flexibility required to solve many of the problems facing the power delivery industry. From conceptual view-point, the UPFC is a generalized synchronous voltage source, represented at the fundamental frequency by voltage $V s l$ with controllable magnitude $(0 \leq V s 1 \leq V s 1$ max $)$ and angle $(0 \leq \varphi s 1 \leq 2 \pi)$ in series with the transmission line. As far as construction is concerned a UPFC consists of shunt (exciting) and series (boosting) transformer, which are connected by two voltage-sourced converters using GTO thyristors valves and a DC circuit. Inverter-2 is used to generate a voltage source at the fundamental frequency with variable amplitude $(0 \leq V s 1 \leq V s 1$ max $)$ and phase angle $(0 \leq \varphi s 1 \leq 2 \pi)$, which is added to the $\mathrm{AC}$ transmission line by the series connected booster transformer. As the series transformer injects series voltage in line, the control of active and reactive power is possible by changing the magnitude and angle of inserted voltage. The real power flows from shunt converter to series converter via a DC link. As both inverters are capable of handling reactive power independently shunt transformer can also inject reactive power on the bus thus helps in maintaining better voltage profile. In this way the inverter output voltage injected in series with the line can be used for direct voltage control, series compensation, phase shifting and their combination and shunt current can be used to maintain good voltage profile. The schematic diagram of UPFC is shown in Fig.4

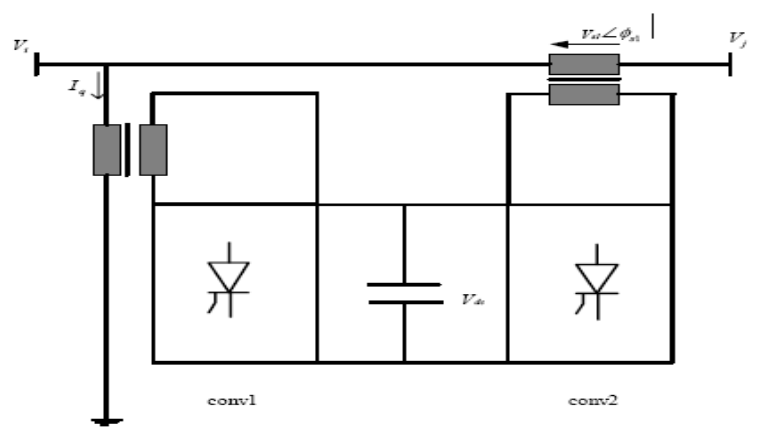

Figure 4 UPFC schematic diagram

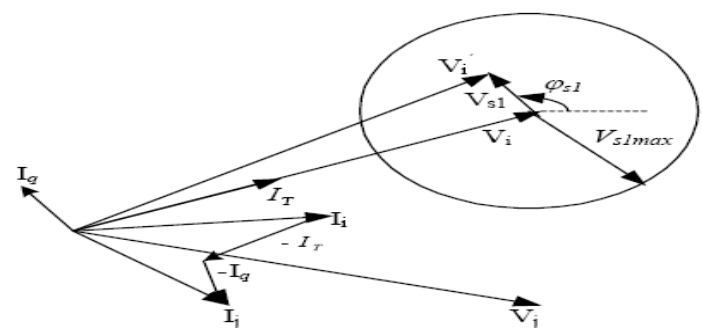

Figure 5 Vector diagram of UPFC

UPFC has three controllable parameters, namely the magnitude and the angle of inserted voltage $(V s 1, \boldsymbol{\Phi} s 1)$ in line- $k$ and the magnitude of the current $(\mathrm{I} q)$. The vector diagram of UPFC is shown in Fig. 5 and circuit diagram is given in Fig. 6. Based on the principle of UPFC operation and the circuit diagram, the basic mathematical relations can be written as:

$$
\begin{gathered}
I_{i j}=\left(V_{i}+V_{s l}-V_{j}\right) y_{i j} \\
\operatorname{Arg}\left(I_{q}\right)=\operatorname{Arg}\left(V_{i}\right) \pm \pi / 2 \\
I_{T}^{*}=\frac{\operatorname{Re}\left[V_{s I} I_{i j}^{*}\right]}{V_{i}}
\end{gathered}
$$

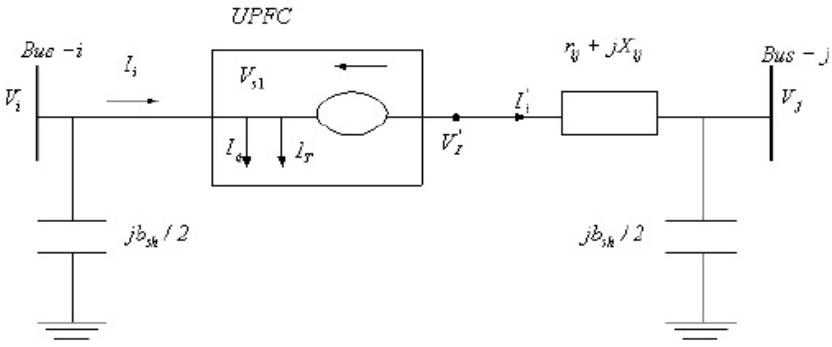

Figure 6 Circuit diagram of UPFC

The power injection at bus- $i$ can be written as:

$$
S_{i}=P_{i}+j Q_{i}=V_{i} I_{i j}^{*}+V_{i}\left(I_{T}+j I_{q}\right)^{*}+\sum_{\substack{i=1 \\ \neq j}}^{n} V_{i} I_{i n}^{*}+V_{i} I_{s h}^{*}
$$

Where, Ish is the shunt current due to line charging.

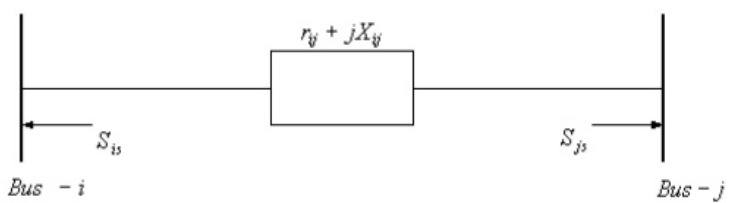

Figure 7 Injection model of UPFC

The effect of UPFC can be represented as injected power with the network as shown in Fig. 7. The injected complex powers Sig $(=P i g+j Q i g)$ at bus- $i$ and $S j g(=P j g+j Q j g)$ at bus-j can be written as,

$$
S_{i g}=S_{i}^{0}-S_{i}=\left\{V_{i} V_{s l}^{*} y_{i j}^{*}+V_{i}\left(I_{T}+j I_{q}\right)^{*}\right\}
$$

Where, $S^{0} \quad$ is the complex power injection when there is no UPFC. From eqn. 8, the real and reactive power injections at bus-i can be derived as

$$
\begin{gathered}
P_{i g}=-\operatorname{Re}\left\{V_{i} V_{s 1}^{*} y_{i j}^{*}\right\}-V_{i} I_{T}^{*} \\
Q_{i g}=-\operatorname{Im}\left\{V_{i} V_{s 1}^{*} y_{i j}^{*}\right\}+V_{i} I_{q}
\end{gathered}
$$


Similarly the real and reactive powers injections at bus- $j$ and bus- $g$ can be derived as

$$
\begin{gathered}
P_{j g}=V_{j} V_{s I}\left(g_{i j} \cos \left(\varphi_{s I}-\delta_{j}\right)-b_{i j} \sin \left(\varphi_{s I}-\delta_{j}\right)\right) \\
Q_{j g}=-V_{j} V_{s l}\left(g_{i j} \sin \left(\varphi_{s I}-\delta_{j}\right)+b_{i j} \cos \left(\varphi_{s I}-\delta_{j}\right)\right)
\end{gathered}
$$

\section{OPTIMAL LOCATION FOR TCSC \& UPFC}

Even though FACTS devices offer many advantages, their installation cost is very high. Hence ISO has to locate them at optimal locations. This task can be accomplished by considering many factors like cost, thermal limits of transmission lines, reactive power compensation, reduction of system losses, voltage limits and stability limits. As the aim of this paper is to relieve the congestion here considering line loading optimal locations are obtained [18]-[22].

\subsection{Objectives of Optimization}

The objective function is built in order to penalize the configurations of FACTS leading to overloaded transmission lines. Only the technical benefits of the FACTS controllers, in terms of loadability, are taken into account. Therefore, for configuration of FACTS devices, the objective function to maximize is given by,

$$
C_{f g}=\Pi_{\text {line }=1}^{N_{L}} O v l_{\text {line }}
$$

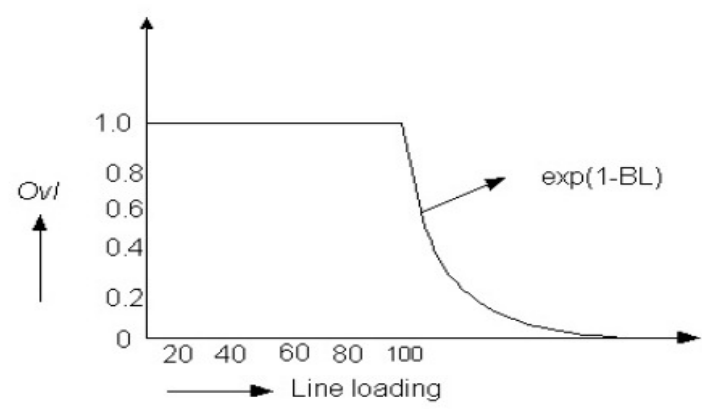

Figure 8 Objective function vs line overload.

While the branch loading is less than $100 \%$, its value is equal to 1 ; then it decreases exponentially with the overload. To accelerate the convergence, the product of all objective function is taken. Equation (24) is solved using GA with the initial population for a given power system of $\mathrm{Nb}$ branches, is generated from the following parameters:

- $N F$ the number of FACTS devices to be located optimally.

- the different types of devices to be located.

- $N v$ the number of possible discrete setting for a device.

- $N i$ the number of individuals of the population.

The creation of an individual is done in three stages. First, a set of $N F$ branches of the network are randomly drawn and is put in the first string. The order of the branches is not important and different individuals may represent the same configuration of FACTS devices. After drawing the branches where the FACTS devices will be located, the next two steps consist in the attribution of the characteristics of the devices. The second string, referred to the types of the devices, is obtained by randomly drawing numbers among the selected devices. Thus, if we decide to optimally locate only one type of device, this string will contain the same character. Setting values of the devices are finally randomly drawn among the possible. To obtain the entire initial population, these operations are repeated $\mathrm{Ni}$ times.

Then, the objective function (Eqn (24)) is computed for every individuals of the population. It represents a mathematical translation of the optimization to realize and does not have to be continuous or derivable. It has to be elaborated so as to favor the reproduction of good individuals without preventing reproduction of interesting other. In our case, the objective function is defined in order to quantify the impact of the FACTS devices on the state of the power system. The move to a new generation is done from the results obtained for the old generation. A biased roulette wheel is created from the obtained values of the objective function of the current population as represented in Fig. 9.

After that, the operators of reproduction, crossover and mutation are applied successively to generate the offspring. In turn, two individuals are randomly drawn from the population and reproduced. The probability of drawing an individual is proportional to its part on the biased roulette wheel. Fig. 9(a) shows the process of reproduction. The crossover may occur with a probability; generally close to 1. A single-point crossover is applied as shown in Fig. 10. From the position of crossover point, elements of the three strings of both parents are exchanged.

Mutations are possible independently on all elements of the three strings of an individual. A specific probability is applied for each string: for the first string, for the second and for the last. These probabilities change with the generations. When a mutation occurs on the first string, the one related to the location, a new line among the set of branches having no FACTS is randomly drawn. In the case of mutation on the two other strings, a new value is drawn among the set of possible ones. Examples of mutations are shown in Fig. 11

Operations of selection, crossover and mutation are repeated until the number of desired offsprings is created. The objective function is then calculated for every offsprings and the best individuals among the entire pool, comprising parents and their offsprings, are kept to constitute the new generation.. 

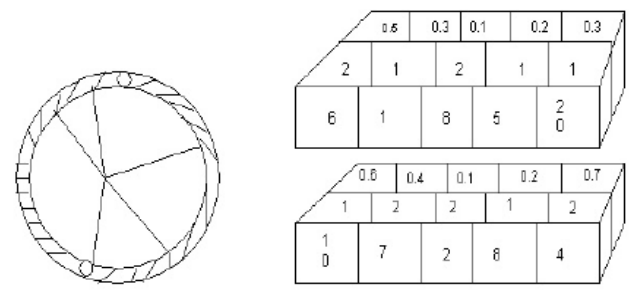

Figure 9 Reproduction (a) Draws on the roulette wheel (b) Selected individuals.
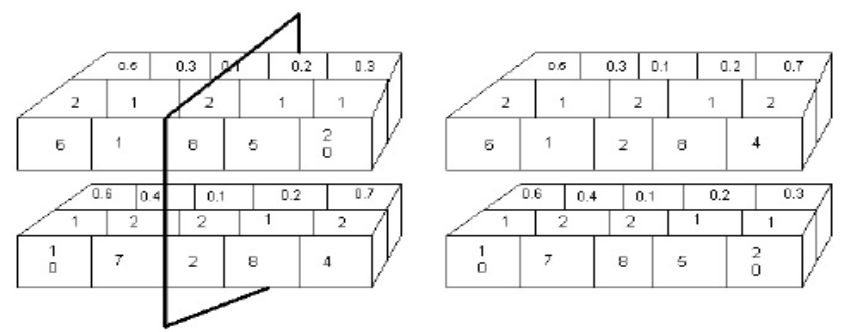

Figure 10 Cross over (a) Cross over point (b) After crossover.

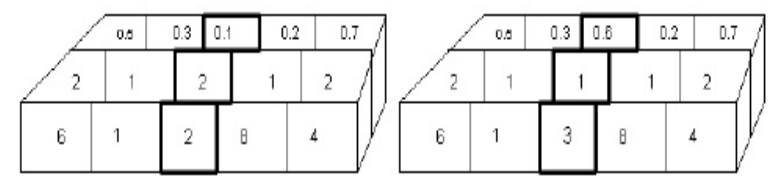

Figure 11 Mutation (a) Mutation point (b) After mutation.

In this way, the objective function of the best individual of the new generation will be the same or higher than the objective function of the best individual of the previous generation. Similarly, the average fitness of the population will be the same or higher than the average fitness of the previous generation. Thus the fitness of the entire population and the fitness of the best individual are increasing for each generation. The termination criteria for GA will be any one of the following two conditions:

(1). The maximum number of generations is achieved.

(2). When the genotype of the population of individuals converges, the convergences of the genotype structure occur when all bit positions in all string are identical. In this case, crossover will have no further effect.

\section{RESULTS AND DISCUSSIONS}

The proposed model has been implemented on IEEE57 bus system. The social welfare given in equation (1) is taken as Objective function for congestion management. This objective function is solved using Genetic Algorithm. The parameters used for GA is given in Table 1 .
Table 1. Genetic Algorithm Parameters

\begin{tabular}{|l|l|}
\hline Population size & 150 \\
\hline Mutation rate & 0.01 \\
\hline Crossover rate & $100 \%$ \\
\hline Crossover operator & Single point \\
\hline Selection operator & Roulette wheel \\
\hline Maximum iterations & 50 \\
\hline
\end{tabular}

The optimal generation schedule for maximizing social welfare with and without transaction for fixed load is given in Table 2. A bilateral transaction of $100 \mathrm{MW}$ is considered between supplier at node 5 and consumer at node 15 . The generation cost, customer benefit and social welfare for elastic and inelastic loads are given in the Table 3.

Table 2. Optimal Generation schedule

\begin{tabular}{|c|c|c|c|c|c|}
\hline & \multicolumn{2}{|c|}{ Inelastic load } & \multicolumn{3}{|c|}{ Elastic load } \\
\hline & 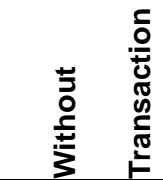 & 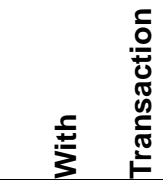 & 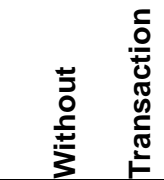 & $\stackrel{5}{\xi}$ & 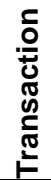 \\
\hline $\begin{array}{l}\text { Total } \\
\text { generation } \\
\text { cost }\end{array}$ & 11320 & 13470 & 6160 & 8908 & \\
\hline $\begin{array}{l}\text { Customer } \\
\text { benefit }\end{array}$ & 5902 & 7309 & 3663 & 4895 & \\
\hline Social welfare & 5417 & 6162 & 2497 & 4013 & \\
\hline $\begin{array}{l}\text { Total } \\
\text { generation }\end{array}$ & 428.8 & 528.8 & 263.3 & 352.9 & \\
\hline Total load & 428.8 & 5288 & 263.2 & 352.9 & \\
\hline
\end{tabular}


Table 3 Optimal social welfare

\begin{tabular}{|c|c|c|c|c|}
\hline \multirow[b]{2}{*}{$\begin{array}{l}0 \\
2 \\
\vdots \\
\frac{1}{0} \\
\frac{\pi}{2} \\
0 \\
0 \\
0 \\
0\end{array}$} & \multicolumn{2}{|c|}{ Inelastic load } & \multicolumn{2}{|c|}{ Elastic load } \\
\hline & 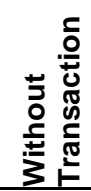 & 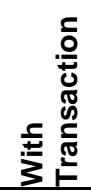 & 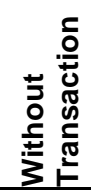 & 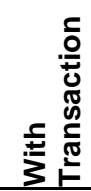 \\
\hline 1 & 0 & 0 & 0 & 0 \\
\hline 2 & 0 & 0 & 0 & 0 \\
\hline 3 & 19.1 & 34.82 & 13.18 & 13.88 \\
\hline 4 & 0 & 0 & 0 & 0 \\
\hline 5 & 277.1 & 378.4 & 152.4 & 246.7 \\
\hline 6 & 0 & 0 & 0 & 0 \\
\hline 7 & 132.7 & 115.5 & 97.69 & 95.18 \\
\hline
\end{tabular}

\section{Without transaction}

The fig. 12 shows the convergence criteria of the objective function in maximizing the social welfare without transaction. The line loading without transaction is given in the fig. 13

\section{With Transaction}

The figure 14 shows the convergence criteria of the objective function is maximizing the social welfare for without transaction. The line loading without Transaction are given in the fig.16. It is found that the line number 8 (connected between buses 5 and 6) is congested for a bilateral Transaction of $100 \mathrm{MW}$. The line thermal limit is taken $1.5 \mathrm{pu}$

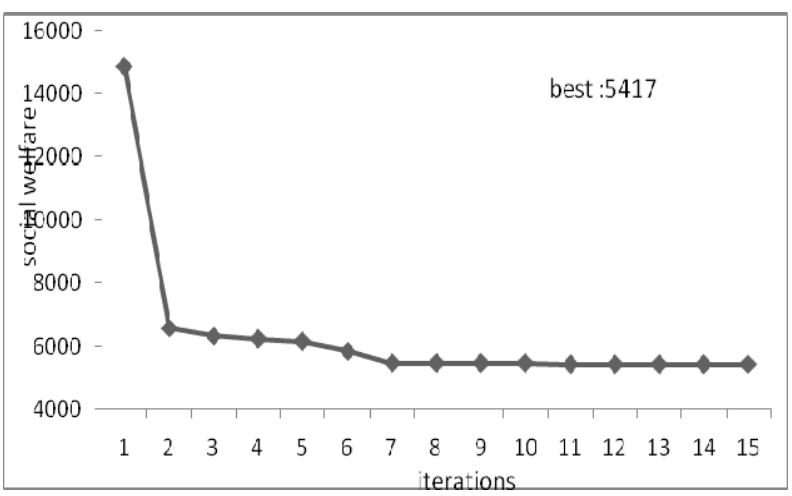

Figure 12 Social welfare vs iterations without transaction

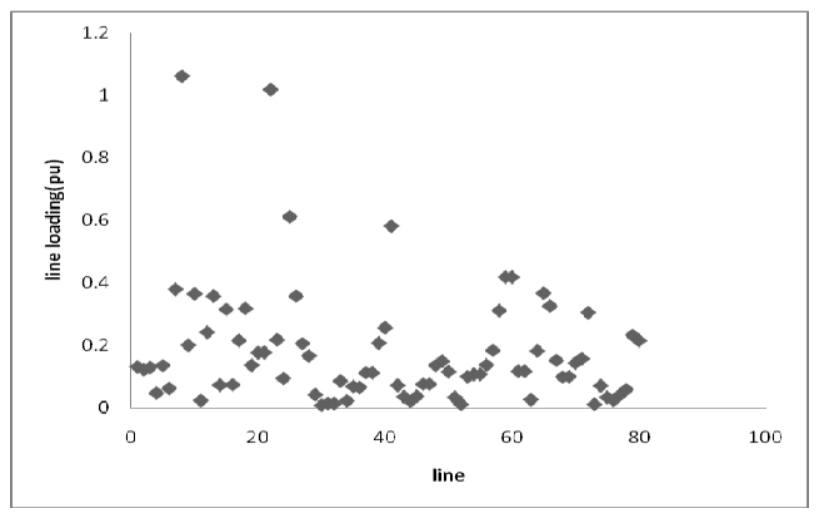

Figure 13 Line loading without transaction

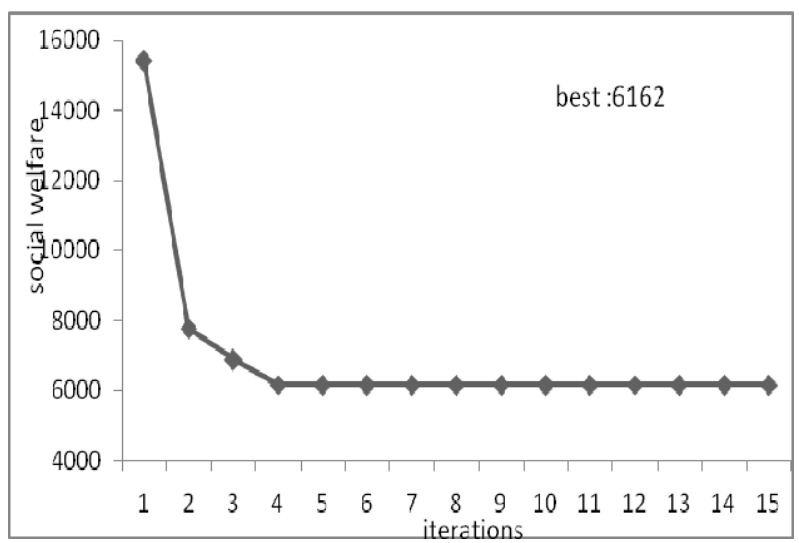

Figure 14 Social welfare vs iterations with transaction

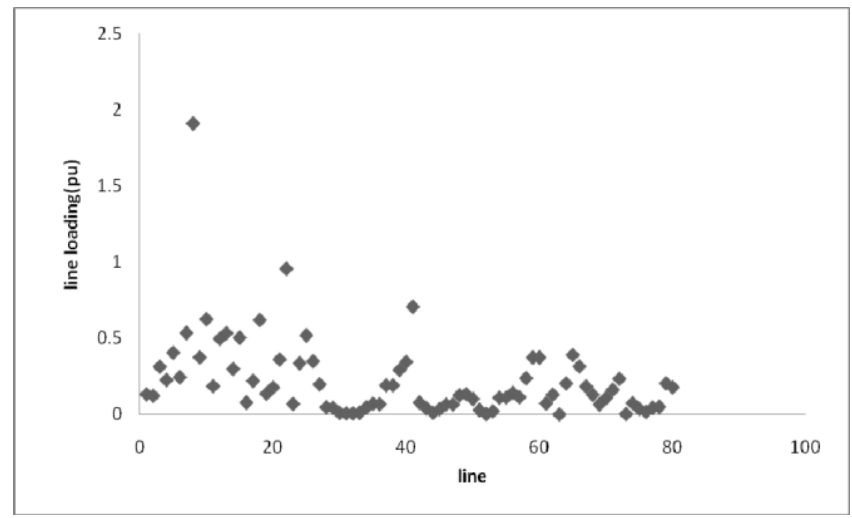

Figure 15 Line loading without transaction 


\section{Using FACTS Controller:}

To relive congestion FACTS Controllers TCSC and UPFC are considered. To find the optimal location of the FACT controllers, the objective function Eqn 4.14. is solved using Genetic Algorithm. The convergence of the objective function is given fig. 16 and fig. 18

Two cases are considered

\section{Locating Three TCSC}

\section{Locating Three UPFC}

\section{Case 1 - Three TCSC}

The optimal location of TCSC in the lines 15,16,19 are obtained using Genetic Algorithm. It is found that while using TCSC, the congestion in the line 8 is relieved from $1.9 \mathrm{pu}$ to $1.45 \mathrm{pu}$. The line loading with three TCSC is shown in fig. 17.

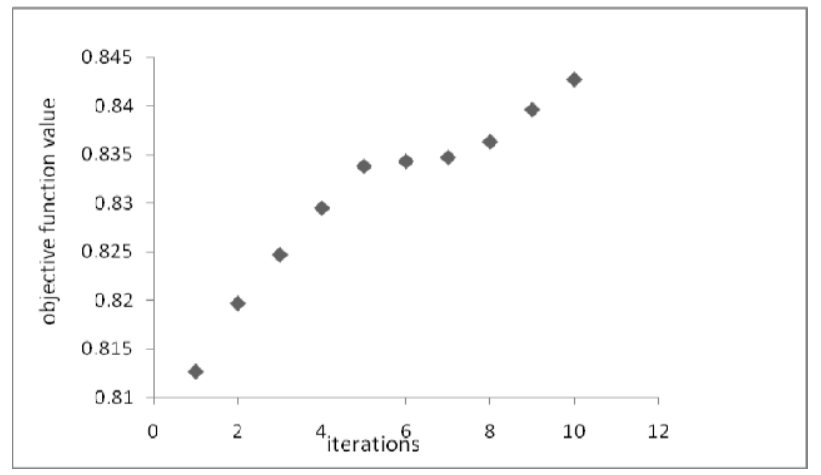

Figure 16 Ovl line vs. iterations with Three TCSC Best: 0.8427

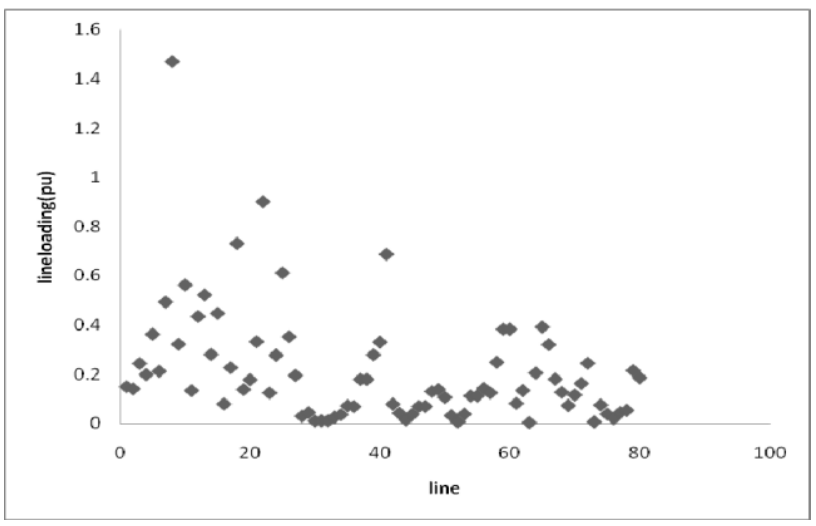

\section{Figure 17 Line loading with TCSC}

\section{Case 2 - Three UPFC}

The optimal location of UPFC in the lines 15, 16, 19 are obtained using Genetic Algorithm. It is found that by locating UPFC, the congestion in the line 8 is relieved from $1.9 \mathrm{pu}$ to $1.37 \mathrm{pu}$. The line loading with three UPFC is shown in figure 19. The Line loading reduction for locating three UPFCs is more as compared with three TCSC.

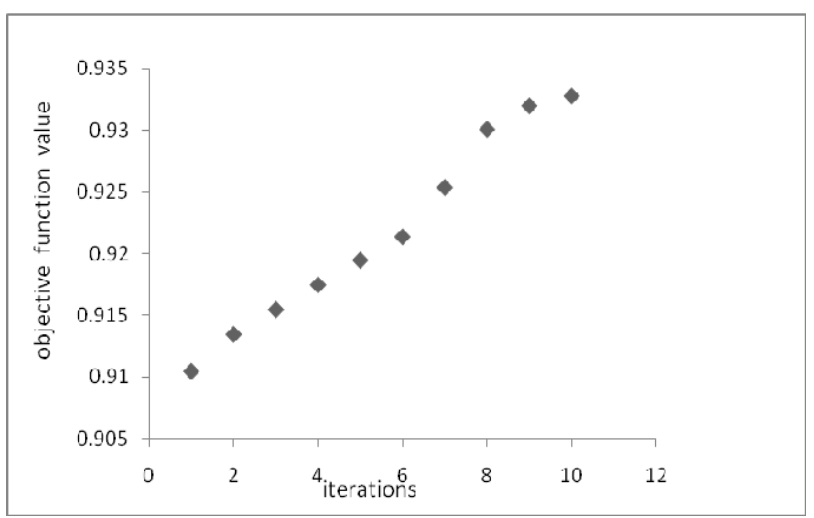

Fig. 18 Ovl line Vs. iterations with three UPFC Best: 0.932

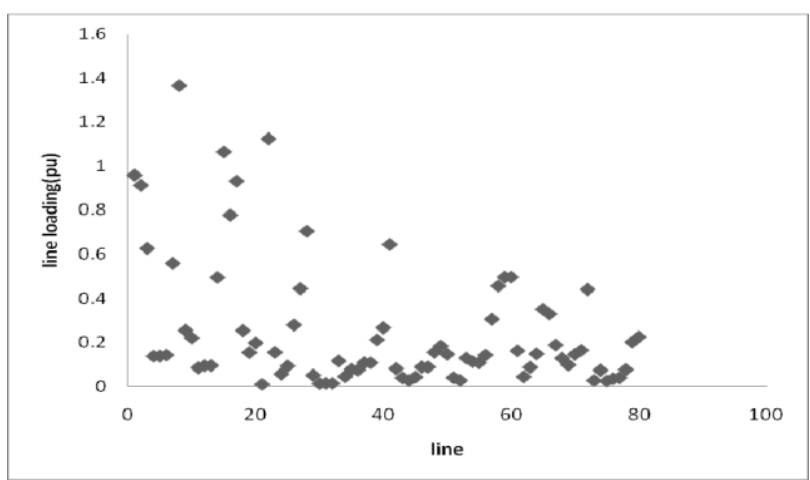

Figure 19 Line loading with UPFC

\section{CONCLUSIONS}

In this paper an algorithm for congestion management using OPF has been proposed and it is solved using GA to find the global optimal generation schedule for maximizing the social welfare. By introducing a transaction it is found that the line loading was increased, i.e congested. To relieve congestion multiple types of FACTS devices are located optimally by considering thermal limits of the lines.

Analysis is carried out by assuming two types of FACTS devices. Line loading reduction in case of three UPFCs is more as compared to three TCSCs. Above method is tested on IEEE57 bus system and it can be readily extended to any practical network.

In future other FACTS devices can be used for relieving congestion. Social welfare maximization and line overloading problem are solved separately in this paper. Both objectives can be solved simultaneously using any multi objective optimization techniques.

\section{ACKNOWLEDGMENTS}

My thanks to the experts who have contributed towards development of this paper. 


\section{REFERENCES}

[1] R. S. Fang and A. K. David, May 1999. "Optimal Dispatch under transmission contracts," IEEE Transactions on Power Systems, vol. 14, No.2, pp.732-737.

[2] Parveen Kumar and S. C. Srivastava, Dec.2000 "Congestion management in deregulated market $-\mathrm{A}$ case study on an Indian power system," in Proc. National Power Systems Conference, Bangalore, India, pp.191-196.

[3] Richard D. Christie, F. Wollenber, and Ivar Wangensteen, Feb.2000 "Transmission management in the deregulated environment," Proceedings of IEEE, vol. 88, No.2, pp.170-195.

[4] A. K. David, Feb. 1998 "Dispatch methodologies for open access transmission systems," IEEE Transactions on Power Systems, vol. 13, No. 1, pp 46- 53.

[5] R. S. Fang, and A. K. David, August 1999 "Transmission congestion management in electricity market," IEEE Transactions on Power Systems, vol. 14, No. 3, pp.877-883.

[6] Harry Singh, Shan You, and Alex Papalexopalos, May 1998 "Transmission congestion management in competitive electricity market," IEEE Transactions on Power Systems, vol. 13, No. 2, pp.672-680.

[7] H. C. Leung and T. S. Chung, Jan. 2000 "Optimal power flow with a versatile FACTS controller by genetic algorithm approach", in Proc. Power engineering society winter meeting, 2000, vol.4, pp. 2806-2811.

[8] T. S. Chung, and Y. Z. Li, February 2001 "A hybrid GA approach for OPF with consideration of FACTS devices", IEEE Power Engineering Review, pp. 47-50.

[9] P. Bhasaputra and W. Ongasakul, "Optimal power flow with multi-type of FACTS devices by hybrid TS/SA approach", in Proc. IEEE ICIT 2002, Bangkok, Thailand, pp.285-288.

[10] N. G. Hingorani and L. Gyugyi, 1998,Understanding FACTS, The Institution of Electrical and Electronics Engineers.

[11] Y. H. Song, J. Y. Liu, and P. A. Mehta, September 1999. "Power injection modeling and optimal multiplier power flow algorithm for steady-state studies of unified power flow controllers", Electric Power Systems Research, vol. 52, No. 3, pp. 51-59.
[12] L. Gyugyi, July 1992 "A unified power flow control concept for flexible ac transmission systems", IEE Proc., Part-C, vol. 139, No. 4, pp. 323-333.

[13] L. Gyugyi, T. R. Rietman, A. Edris, C. D.S Chauder, D. R. Torgerson, and S. L. Williams, 2001, "The unified power flow controller: a new approach to power transmission control," IEEE Transactions on Power Delivery, vol. 10, No.2, pp. 1085-1097.

[14] C. R. Fuerte, E. Acha, Jan. 1997 “A Newton-type algorithm for the control of power flow in electrical network”, IEEE Transaction on Power Systems, vol. 12, No. 4, pp. 1474-1480.

[15] A. G. Bakirtzis, Pandel N. Biskas, Christoforous E. Zoumas, and Vasilios Petridis, May 2002 "Optimal power flow by genetic algorithm", IEEE Transactions on Power Systems, vol. 17, No. 2, pp.229-236.

[16] Ying Xiao, Y. H. Song, and Y. Z. Sun, November 2002 "Power flow control approach to power systems with embedded FACTS devices", IEEE Transactions on Power Systems, vol. 17, No. 4, pp. 943949.

[17] M. H. Haque, and C. M. Yam, September 2003 "A simple method of solving the controlled load flow problem of a power system in the presence of UPFC," Electric Power Systems Research, vol. 65, No.3, pp.55-62.

[18] Stephane Gerbex, Rachid Cherkaouri, and Alain J. Germond, Aug-2001 "Optimal location of multi-type FACTS device in a power system by means of genetic algorithms", IEEE Transactions of Power Systems, vol. 16, No. 3, pp.537-544.

[19] S. N. Singh, and A. K. David, September 2000 "Congestion management by optimizing FACTS devices location," IEEE Power Engineering Review, pp. 58-60.

[20] S. N. Singh, and A. K. David, July 2001 "Optimal location of FACTS devices for congestion management", Electric Power Systems Research, vol. 58, No. 2, pp. 71-79.

[21] T. T. Lie, and W. Deng, 1999 "Optimal Flexible AC Transmission Systems (FACTS) devices allocation," International Journal of Electrical Power and Energy Systems, vol.19. No. 2, pp. 125-134.

[22] K. S. Verma, S. N. Singh and H. O. Gupta, July 2001 "Optimal location of UPFC for congestion management," Electric Power Systems Research, vol. 58, No. 2, pp. 89-96. 